\title{
IS NIPPLE-SPARING MASTECTOMY AN OPTION FOR PATIENTS WITH IPSILATERAL BREAST CANCER RECURRENCE?
}

\author{
Antônio Luiz Frasson"1, Martina Lichtenfels², Fernanda Barbosa ${ }^{1}$, Alessandra Borba Souza², Carolina Malhone \\ ${ }^{1}$ Hospital Israelita Albert Einstein - Porto Alegre (SP), Brazil. \\ ${ }^{2}$ Hospital São Lucas, PontifíciaUniversidade Católica - Porto Alegre (RS), Brazil.
}

Introduction: Nipple-sparing mastectomy (NSM) is a conservative mastectomy approach for breast cancer with oncological safety and good aesthetic satisfaction. The initial indications of NSM excluded patients with previous radiation, ptosis, high body mass index (BMI) and macromastia, although these contraindications have been challenged. Different authors expanded the classic indications of NSM for patients with previous breast surgery/irradiation, neoadjuvant chemotherapy, and short tumor-nipple distance and showed safety and low complication rates. However, there are few data about the suitability of performing NSM with immediate reconstruction for the treatment of recurrent disease. Objectives: The aim of this study was to analyze the complication and local recurrence rates of 24 patients with indication of mastectomy, however with no skin or nipple involvement, who opted to undergo NSMs with immediate reconstruction for the treatment of ipsilateral breast tumor recurrence (IBTR). Methods: Between January 2001 and December 2018, we evaluated 24 patients that underwent NSMs for the treatment of IBRT after a previous BCS (breast cancer surgery) followed by radiotherapy. All patients were operated by the same surgeon, the data were retrospectively evaluated by the medical chart and the patient's follow-up was updated during the appointments. Results: The patient's mean age in the salvage surgery was 49 years. The majority of patients (70.8\%) underwent bilateral procedures, one (5.9\%) patient due to the diagnosis of invasive cancer in both breasts, one (5.9\%) DCIS in both breasts, and $15(88.2 \%)$ patients without disease in the contralateral breast, most of them for prophylactic or aesthetic reasons to avoid asymmetry and to reach a better aesthetic result. Breast reconstruction was performed using silicone prosthetic implants for 22 (91.7\%) patients and with tissue expander for only two (8.3\%) patients. Patients were followed for a mean time of 132 months since the first surgery. In the mean follow-up of 40 months after the NSM surgery, five (20.8\%) patients were diagnosed with local recurrence and only one patient died from the progression of the disease. Patients presented $4.8 \%$ of partial and $2.4 \%$ of total nipple necrosis. No infection and no implant loss was observed. Conclusions: In the long-term follow-up since the first surgery, we observed low complication rates and a good survival rate, although associated with high local recurrence in patients diagnosed with IBTR undergoing NSM as salvage surgery. We demonstrated that NSM may be considered after IBTR for patients with indication of mastectomy, with no skin or NAC (nipple-areola complex) involvement, who did not want to undergo total mastectomy with resection of the skin and NAC. 